Annuaire du Collège de France 2017-2018

\title{
Chimie du solide et énergie
}

Jean-Marie Tarascon

\section{(2) OpenEdition}

Journals

Édition électronique

URL : https://journals.openedition.org/annuaire-cdf/15703

DOI : 10.4000/annuaire-cdf.15703

ISBN : 978-2-7226-0572-5

ISSN : 2109-9227

Éditeur

Collège de France

Édition imprimée

Date de publication : 30 décembre 2020

Pagination : 83-98

ISBN : 978-2-7226-0516-9

ISSN : 0069-5580

Référence électronique

Jean-Marie Tarascon, « Chimie du solide et énergie », L'annuaire du Collège de France [En ligne], 118| 2020, mis en ligne le 01 avril 2021, consulté le 22 août 2022. URL : http://journals.openedition.org/ annuaire-cdf/15703 ; DOI : https://doi.org/10.4000/annuaire-cdf.15703 


\title{
CHIMIE DU SOLIDE ET ÉNERGIE
}

\author{
Jean-Marie TARASCON \\ Membre de l'Institut (Académie des sciences, \\ professeur au Collège de France
}

Mots-clés : chimie, énergie, stockage de l'énergie, batterie

La série de cours et séminaires «Électrochimie appliquée : rôle des électrolytes et interfaces pour dispositifs de stockage et conversion » est disponible, en audio et/ou vidéo, sur le site du Collège de France (https://www.college-de-france.fr/site/jeanmarie-tarascon/course-2017-2018.htm).

\section{ENSEIGNEMENT}

COURS ET SÉMINAIRES - ÉLECTROCHIMIE APPLIQUÉE : RÔLE DES ÉLECTROLYTES ET INTERFACES POUR DISPOSITIFS DE STOCKAGE ET CONVERSION

Dans le cadre du contexte énergétique, le stockage électrochimique de l'énergie joue un rôle clé, et ce afin de faciliter la mobilité électrique, ainsi que le développement des énergies renouvelables. Ce secteur est en émulation constante avec des avancées notoires répétitives. J'avais précédemment traité certaines de ces avancées au travers des technologies Li-ion, Na-ion, $\mathrm{Li}(\mathrm{Na})$-air, $\mathrm{Li}-\mathrm{S}$, voire des technologies à ions multivalents $(\mathrm{Ca}, \mathrm{Mg})$. Un dénominateur commun à toutes ces technologies est l'électrolyte, qui revêt une importance capitale car il détermine la plage de potentiels d'opération de la batterie et donc sa densité d'énergie si l'on en maîtrise sa stabilité thermique et électrochimique. Sont associés intimement aux électrolytes les problèmes d'interfaces, qui sont à l'origine de la défaillance des accumulateurs notamment en termes de sécurité, durabilité et vie calendaire. Elles sont le cauchemar de l'électrochimiste. Ce sont donc ces deux aspects qui ont été traités dans ce cours pour poser les bases fondamentales pour la compréhension des technologies existantes, mais aussi pour décrire des technologies en réémergence telles les batteries à flux circulants (redox flow) et à électrolyte tout solide, qui sont aujourd'hui très médiatisées. 
Ce cours a été doublé par des exposés portant sur d'autres systèmes de stockage et conversion qui incluront le stockage capacitif, l'électrocatalyse, et ce afin d'illustrer la complexité à maîtriser les interfaces malgré le développement de nouvelles techniques d'analyse, dont certaines seront décrites. La retombée de ces recherches pour l'industrie et l'économie de demain, notamment dans le domaine du véhicule électrique, a également été abordée. Chacun de ces séminaires a été tenu par des experts venant de différents secteurs universitaires (Bordeaux, Toulouse, Rennes, Amiens, Paris) et du secteur industriel pour l'un d'entre eux (IFP).

\section{Cours 1 - Des bases fondamentales au choix de solvants et sels appropriés pour la conception de formulation d'électrolytes performants}

\section{2 février 2018}

Un bref rappel historique a introduit les premières observations de conduction ionique dans les solides par Michaël Faraday en 1834 et a mentionné la théorie de dissociation électrolytique proposée en 1884. Les électrolytes liquides, qui sont des substances faisant apparaître des ions entourés d'une sphère de solvatation, ont été par la suite présentés tout en définissant certains des paramètres physico-chimiques clés qui les caractérisent. C'est ainsi que la mobilité $\left(\mu_{\mathrm{i}}\right)$ d'un ion, son nombre de transport $\left(\mathrm{t}_{\mathrm{li}+}\right)$ ont été définis tout autant que la constante diélectrique d'un solvant $(\varepsilon)$ qui contrôle son pouvoir dissociant ainsi que sa viscosité $(\eta)$. L'effet ambivalent de ces deux derniers paramètres sur la conductivité ionique d'un électrolyte (soluté + solvant) a été illustré et le phénomène néfaste d'agrégation d'ions sur la conductivité, décrit. Fort de ces notions fondamentales pour l'établissement du cahier des charges d'un solvant et d'un soluté idéal, le cheminement intellectuel conduisant à la confection des électrolytes actuels utilisés dans les batteries à ions $\mathrm{Li}$ a été détaillé. L'électrolyte magique à ce jour, qui repose principalement sur l'utilisation d'un sel de $\mathrm{Li}\left(\mathrm{LiPF}_{6}\right)$, dont l'anion possède une charge délocalisée pour être moins réactif et est dissous dans un mélange de solvants aprotiques polaires tels des carbonates cycliques et acycliques, a été présenté. Il a également été fait état des recherches actuelles visant à l'élaboration de meilleurs sels et meilleurs solvants. Ces recherches intègrent bien évidemment la compatibilité électrochimique de ces électrolytes avec les matériaux d'électrodes, en y introduisant des notions de stabilité thermodynamique des électrolytes via la détermination de leurs frontières orbitales HUMO et LOMO. Sur la base de ces considérations, la notion de SEI (solid electrolyte interface en anglais), dont la formation résulte de réactions parasites, a été introduite.

\section{Séminaire 1 - Vers l'électrification de nos véhicules, quels enjeux, quelles solutions?}

François Badin (Filières Industrielles CARNOT, IFP Énergies nouvelles, Direction Systèmes, Moteurs et Véhicules)

Les transports routiers font face ces dernières années à de multiples enjeux, principalement :

1. niveau global : l'objectif est de réduire les émissions de gaz à effet de serre, la part des transports routiers étant de l'ordre de $30 \%$ au total en France ;

2. niveau local : l'objectif est de réduire ou d'éliminer les nuisances locales comme la pollution atmosphérique ou le bruit à basse vitesse en ville. 
L'électrification des transports routiers est une solution qui permet d'agir à différents niveaux sur ces deux contraintes. Les solutions proposées par les constructeurs couvrent un panel très large d'architectures, certaines sont très proches de l'existant comme les Stop \& Start, d'autres sont plus en rupture comme les hybrides rechargeables ou les électriques.

La réalisation d'un bilan environnemental de ces solutions est complexe, car il faut considérer le véhicule (fabrication, maintenance, fin de vie) et l'énergie consommée. Celle-ci peut être un hydrocarbure (véhicule conventionnel et hybride), de l'électricité (véhicule électrique), les deux combinés (véhicule hybride rechargeable) ou même de l'hydrogène (véhicule à pile à combustible).

L'objet de la conférence est de réaliser un panorama de ces solutions d'électrification en faisant apparaître les spécificités et les enjeux qui sont liés.

\section{Cours 2 - Formation et croissance des interfaces électrodes/électrolytes (SEI) au sein des batteries : leurs rôles et complexités avec quelques possibilités de les contrôler}

\section{9 février 2018}

L'aspect thermodynamique des batteries à ions Li sera exposé pour mettre en exergue l'origine de la formation des réactions parasites conduisant à la formation d'interfaces solides (SEI) aux électrodes positive et négative; cet aspect sera d'autant plus propice que le potentiel redox des électrodes positives et négatives se trouve en dehors de la stabilité thermodynamique de l'électrolyte. À titre illustratif, nous avons montré que ce scénario n'est pas unique, en rappelant également que la technologie acide-plomb délivre un potentiel de 2,2 V bien qu'utilisant un électrolyte, l'eau, dont le potentiel thermodynamique n'est que de $1,2 \mathrm{~V}$. De tels systèmes sont donc sous contrôle cinétique avec, pour le plus grand bénéfice de la technologie Li-ion, la formation d'une SEI protectrice qui évolue peu avec le temps en raison d'une cinétique lente des réactions parasites. Nous avons mis en évidence que la formation d'une SEI auto-passivante, constituée principalement de $\mathrm{LiCl}$, est à l'origine de la batterie primaire $\mathrm{Li}-\mathrm{SOCl}_{2}$ : sans formation de cette SEI la technologie Li-ion n'existerait pas. Bien que pouvant être considérée comme un don de la nature, il n'empêche que cette SEI demeure un cauchemar à maîtriser pour les scientifiques. Il s'agit en effet d'une interface dynamique, dont nature, composition et densité changent en fonction de l'électrolyte (solvant + sel) et du matériau d'électrode. Nous avons montré que malgré plus de 25 ans de recherches, le mystère de la formation, la nature et la composition de la SEI n'est pas encore totalement levé malgré le développement de techniques analytiques in situ puissantes telles la résonance magnétique nucléaire, la spectroscopie photoélectrique à rayonnement $\mathrm{X}$, voire la microscopie en mode balayage ou en transmission. Un exemple de cette complexité de la SEI sera illustré par le biais de la réaction d'insertion du $\mathrm{Li}^{+}$dans les carbones graphitiques avec l'interposition de réactions de co-intercalation de solvants, voire d'exfoliation. Bien qu'ignorée pendant de nombreuses années, on montrera que cette SEI existe à l'électrode positive avec une complexité analogue à celle des mécanismes actuellement développés. Enfin, nous avons clos ce cours par l'interface électrodes/électrolytes-collecteurs de courant en s'attachant plus particulièrement à la corrosion, l'oxydation anodique et la dissolution de l'Al selon la nature des sels. L'avantage de $\mathrm{LiPF}_{6}$ sur LiTFSI sera ainsi documenté. 


\section{Séminaire 2 - Modification, caractérisation électrochimique des interfaces de carbone}

Philippe Hapiot (directeur de recherche CNRS, Institut des sciences chimiques de Rennes, université de Rennes 1)

Maîtriser, modifier, étudier les propriétés d'une interface sont souvent la clé dans de nombreux problèmes technologiques, que ce soit en rapport avec le stockage ou production de l'énergie, les sciences analytiques, l'électrocatalyse... S'il existe aujourd'hui de très nombreuses méthodes pour fonctionnaliser ou caractériser la surface d'un matériau, l'électrochimie, par sa nature interfaciale, joue un rôle majeur dans ces travaux. Ces dix dernières années ont ainsi vu le développement simultané d'approches fondées sur l'électro-greffage pour ce qui est de modifier la surface d'un matériau et de techniques électrochimiques localisées, comme la microscopie électrochimique à balayage (SECM), en ce qui concerne la caractérisation de ces modifications.

Dans cet exposé, ces approches analytiques et leurs principes sont présentés en s'appuyant sur des exemples qui concernent notamment la fonctionnalisation des interfaces de carbone par électro-greffage, tout en précisant les avantages et difficultés par rapport à d'autres techniques classiques. Les réactions d'électrogreffage impliquent généralement la production électrochimique d'un intermédiaire hautement réactif à partir d'un précurseur électro-actif qui va réagir avec la surface. Les avantages sont à la fois une fixation robuste sur une large gamme de matériaux, notamment le carbone avec la simplicité de la préparation de précurseurs peu coûteux tels les sels d'aryldiazonium. La microscopie électrochimique fournit quant à elle une véritable vision chimique et dynamique de la surface modifiée, car l'interface est vue au travers de l'interaction entre une molécule redox produite à une microélectrode et l'échantillon à étudier.

\section{Cours 3 - De l'enrobage de particules à l'utilisation d'additifs à fonctions variées pour contrôler la SEI au niveau des électrodes}

\section{6 février 2018}

La SEI gouverne la performance des accumulateurs en termes de cyclage, puissance, durabilité et vie calendaire. Il nous appartient donc de maîtriser sa formation et d'en contrôler son évolution, et ce à différentes températures. Dans la quête vers une SEI idéale, les recherches actuelles visent soit à agir au niveau de l'électrode via l'utilisation d'enrobage des particules et des méthodes de traitement de surface par voie solution ou céramique, soit à agir au niveau des électrolytes via l'utilisation d'additifs qui se décomposeront électrochimiquement à des potentiels compris dans la fenêtre de potentiels thermodynamiques de l'électrolyte permettant de faire sur mesure une SEI protectrice. Nous avons ainsi passé en revue les méthodes de traitement de surface avant d'aborder la science des additifs. On a tenté de classifier cette vaste quantité d'additifs selon leur principe de fonctionnement, qui peut reposer soit sur la polymérisation de monomères induite électrochimiquement, soit sur la décomposition réductive d'hétéroélements (ex : S) à haut état d'oxydation, voire de molécules à base de $\mathrm{F}, \mathrm{Cl}$, etc. conduisant à la formation d'une couche protectrice ( $\mathrm{LiF}, \mathrm{LiCl}$ ou autre). Enfin, avons montré l'importance des additifs à triple liaison $(-\mathrm{C} \equiv \mathrm{N})$ et leur synergie avec ceux à double liaison $(-\mathrm{C}=\mathrm{C}-)$, ainsi que l'importance de l'enrobage, via la 
décomposition in situ d'additifs de type tris(trimethylsilyl)phosphate (TMSP), pour la protection d'oxydes à haut potentiel $\left(\mathrm{LiCoO}_{2}, \mathrm{LiMn}_{2} \mathrm{O}_{4}, \ldots\right)$ dont nous en avons mentionné les sites actifs déduits par des mesures de XPS. Au travers de ce parcours, il en ressortira que l'ajout d'additifs est fondé sur une science d'essais et d'erreurs, avec cependant la possibilité d'extraire certaines tendances qui se sont avérées très utiles dans la confection rapide d'un électrolyte optimisé pour la technologie Na-ion. Bien entendu, il va de soi que nous n'aurions pu clore cette vaste recherche sans mentionner les travaux actuels dédiés à la recherche de nouveaux additifs par le dépistage haut débit, en prenant comme exemple le «Throughput Screening System » développé à l'université de Münster (Allemagne).

\section{Séminaire 3 - Électrochimie sans fil pour des applications en micro- et nanotechnologie}

Alexander Kuhn (professeur, Institut des sciences moléculaires, CNRS UMR 5255, université de Bordeaux, Bordeaux INP-Aquitaine, groupe Nanosystèmes analytiques)

Une conversion électrochimique s'effectue dans la plupart des cas de manière classique, c'est-à-dire à la surface d'électrodes qui sont connectées à une source d'électricité. Cependant, il existe une voie alternative qui permet de déclencher des processus électrochimiques à distance sur des objets qui ne sont pas en contact physique avec un générateur de tension ou de courant. Cette «électrochimie sans fil », ou plus scientifiquement appelé « électrochimie bipolaire », a un long historique, y compris dans le domaine des batteries. Le concept connaît actuellement une véritable renaissance dans différents domaines scientifiques, notamment à cause des applications potentielles en micro- et nanotechnologie qui ont été mises en lumière pendant la dernière décennie. L'approche permet, entre autres, la modification très contrôlée de surfaces à l'échelle micro- et nanométrique. Dans ce séminaire, nous avons discuté des grands axes de recherche dans ce domaine et illustrer la puissance du concept aussi avec des exemples qui vont bien au-delà de la chimie.

\section{Cours 4 - Des additifs pour la sécurité à l'utilisation de liquides ioniques, ionogels, voire de gaz liquéfiés comme électrolytes}

5 mars 2018

Les additifs sont également essentiels dans l'amélioration de la sécurité des accumulateurs avec notamment l'introduction de navettes redox dont nous avons détaillé le fonctionnement et les limitations, ainsi que des additifs de coupure. Nous avons décrit le cahier des charges pour le choix de ces molécules redox pour contrôler les risques de surcharge et sur-décharge des accumulateurs. Cependant, leur utilisation est limitée à des batteries de portable et non à celles du véhicule électrique, en raison de l'augmentation de température inhérente au mécanisme même de fonctionnement d'une navette. Quant aux additifs de coupure, ils reposent soit sur leur décomposition à haut potentiel, qui conduit à la formation d'un polymère isolant - qui de ce fait empêche l'emballement thermique -, soit sur l'existence d'une transition non métal $\rightarrow$ métal à haut potentiel, si bien qu'une surcharge est empêchée par la création d'un court-circuit interne. Ce procédé permet à un courant de surcharge de passer de manière inoffensive à travers la cellule, offrant ainsi l'avantage d'être réversible. De tels additifs peuvent être ajoutés dans la membrane 
qui joue le rôle de boîte à outils. Cependant, l'approche la plus robuste demeure l'utilisation de séparateurs hétérostructurés à température de coupure ajustable, voire le développement de séparateurs à recouvrement céramique actuellement présents dans des cellules commerciales. Dans la course vers la sécurité, les électrolytes à base de liquides ioniques, voire d'ionogels, ont été présentés. Il s'agit de sels, liquides à température ambiante, constitués de cations et d'anions. Leur conductivité est inversement proportionnelle à la viscosité, selon la règle de Walden dans le cas de liquides ioniques parfaits que l'on caractérise par leur degré d'ionicité, déterminé par des mesures couplées de spectroscopie d'impédance et de RMN qui montrent la concentration d'ions libres (non associés) $v s$ les ions en paires et agrégats. L'injection de liquides ioniques dans des matrices céramiques, voire polymériques ou autres, pour former des membranes flexibles en vue du développement de supercapaciteurs, voire de batteries à performances équivalentes aux systèmes liquides, est un sujet fortement développé tant le caractère non inflammable de ces liquides est attractif.

Nous avons clos enfin la section liquide ionique par les dernières recherches ayant donné naissance à des électrolytes bi-redox, c'est-à-dire des liquides ioniques fonctionnalisés avec des couples redox, qui, de ce fait, ont doté le système d'une capacité excédentaire. Une toute dernière nouveauté réside également dans la mise au point d'électrolytes de gaz liquéfiés (fluorométhane) pouvant fonctionner sur de larges domaines de températures tout en présentant d'excellentes conductivités ioniques que nous évoquerons sur la base de constante diélectrique et viscosité. La pression, associée à l'utilisation des électrolytes liquéfiés, qui peut atteindre des valeurs de $4 \mathrm{MPa}$ à $6 \mathrm{MPa}$, demeure un élément limitant vis-à-vis des applications. Malgré cela, une PME, «South 8 Technologies », a déjà été créée pour exploiter la performance de tels électrolytes dans des supercondensateurs EDLC (carbonecarbone), voire des batteries à ions Li utilisant des boîtiers de cellules renforcés.

\section{Séminaire 4 - Contrôle des interfaces électrochimiques pour l'élaboration de nouveaux électrocatalyseurs : un problème cauchemardesque}

\section{Alexis Grimaud (chargé de recherche CNRS, Collège de France, UMR 8260)}

Avec le besoin toujours plus croissant de systèmes électrochimiques nécessaires afin de stocker les énergies renouvelables, le contrôle des interfaces électrochimiques qui sont au cœur de ces systèmes devient de plus en plus crucial. Cependant, des décennies de développement et d'études fondamentales menées sur les transferts complexes d'ions et d'électrons à ces interfaces solides/solides ou solides/liquides n'ont pas encore permis d'énoncer des règles propres régissant la cinétique de ces échanges afin de les améliorer. Ainsi, la célèbre comparaison de Pauli « God made the bulk, the surface was created by the evil » reste d'actualité, comme illustré par les progrès incessants, durant ces dernières décennies, des performances des batteries Li-ion qui sont gouvernées par des échanges ions/électrons de cœur alors que les progrès dans le développement des électrolyseurs ou piles à combustibles, systèmes gouvernés par des échanges de surface, restent relativement lents.

Dans ce séminaire, nous avons commencé par définir ce que sont les effets électrocatalytiques aux interfaces solides/liquides et avons donné des exemples variés de leur application dans les systèmes de stockage ou de conversion de l'énergie. Nous nous sommes ensuite focalisé sur une réaction cruciale qui est au cœur d'un nombre 
important de ces systèmes électrochimiques : la réaction d'oxydation de l'eau et de dégagement de l'oxygène. En suivant l'évolution dans le temps des différents développements réalisés dans ce domaine, nous nous sommes ensuite efforcés de donner une vision globale des recherches effectuées et de leur débouchés en terme de design de nouveaux électrocatalyseurs. Pour cela, nous avons explicité les forces des différentes approches expérimentales et théoriques utilisées, mais aussi leurs faiblesses. En comparant les échanges ioniques et électroniques se déroulant à ces interfaces solides/liquides aux différents processus biologiques permettant la photosynthèse d'oxygène, nous avons donné, enfin, des pistes de développement futur qui nécessiteront une discussion toujours plus importante entre des domaines jusque-là jugés antinomiques, comme l'électrocatalyse homogène et hétérogène.

\section{Cours 5 - Autres types d'électrolytes à base de solutions aqueuses fortement concentrées en sels (WiSE), de gels, et de polymères}

12 mars 2018

Pour minimiser les implications écologiques associées à l'utilisation des batteries, nous devons choisir des chimies avec un minimum d'empreinte sur la nature. C'est ainsi que des efforts se déploient actuellement sur des électrolytes aqueux avec pour objectif d'agrandir la fenêtre électrochimique de l'eau qui est seulement de 1,23 V, et ce afin d'avoir une alternative moins coûteuse, plus sûre et non toxique aux électrolytes organiques. Ce désir fut réellement réalisé via l'utilisation d'électrolytes aqueux concentrés en sel (24 M Li-TFSI) et dénotés WiSE (Water in Salt Electrolyte), qui permettent de fonctionner à des potentiels supérieurs à $3 \mathrm{~V}$. Ce cours a tenté de mettre en perspective ces avancées sur ces nouveaux électrolytes (e.g. mélange dans lequel le soluté surpasse le solvant en termes de poids et volume). Une revue rapide de la littérature nous a montré que ce concept n'est pas nouveau puisque introduit par A. Angel en 1993 sous la notion de "Polymer in salt», avec par la suite l'observation d'effets bénéfiques en termes de meilleure stabilité cathodique et anodique d'électrolytes non aqueux à forte teneur en sels. Par la suite, on s'est attaché à décrire la science sous-tendant ce phénomène, qui réside dans la modification de la couche interne d'Helmholtz tant en nature qu'en composition. Ainsi, lors de la réduction, les anions TFSI' migrent préférentiellement à l'interface où ils se décomposent, conduisant à la formation d'une couche de $\mathrm{LiF}$ insoluble, repoussant ainsi à un plus bas potentiel la réduction de l'eau. En revanche, l'origine du déplacement simultané du mur d'oxydation vers des potentiels plus élevés reste à ce jour très conversée. Dans la poursuite de ce concept, on a souligné la possibilité de réaliser des cellules de type $\mathrm{LiMn}_{2} \mathrm{O}_{4} / \mathrm{Li}$ aqueuses pouvant fonctionner à $4 \mathrm{~V}$, et ce en combinant un électrolyte aqueux concentré en sel (WiSE) avec un traitement de surface spécifique via le dépôt d'un feuillet amorphe et hautement hydrophobe à base de molécules fluorées immiscibles. Moins médiatisés actuellement, mais plus proches du domaine appliqué, on a mentionné également les électrolytes gélifiés et polymères tout solides. Ces derniers sont à l'origine de la batterie Li-polymère, qui doit fonctionner à $55^{\circ} \mathrm{C}$ en raison de la conductivité ionique limitée de la matrice polymère, et alimente les «Blue Cars », parties intégrantes du projet AUTOLIB. Des améliorations récentes ont été discutées, via l'utilisation de copolymères triblocs sur laquelle l'anion TFSI- est fixé, augmentant ainsi le nombre de transport $\left(t_{\mathrm{Li}+}\right)$, le tout résultant en une meilleure conductivité ionique tout en améliorant les propriétés mécaniques. 


\section{Séminaire 5 - Étude de la réactivité aux interfaces électrochimiques d'électrodes pour le stockage et la conversion d'énergie}

Charles Delacourt (chargé de recherche, Laboratoire de réactivité et de chimie des solides, CNRS, UMR 7314)

Les interfaces électrode-électrolyte constituent le cœur des systèmes électrochimiques de stockage et de conversion d'énergie, tels que les accumulateurs, les piles à combustibles et les électrolyseurs. C'est à ces interfaces qu'ont lieu les réactions électrochimiques sur lesquelles repose le fonctionnement de ces systèmes, via des processus de transfert de charge. En plus des processus électrochimiques principaux, ces interfaces peuvent être le siège de phénomènes parasites conduisant à la passivation ou à l'empoisonnement de l'électrode. La passivation peut être bénéfique - c'est le cas pour les électrodes négatives graphitiques d'accumulateurs à ions lithium -, ou néfaste - par exemple, l'empoisonnement par le monoxyde de carbone à l'anode de la pile à combustible à membrane polymère.

Durant cet exposé, je me suis focalité sur l'étude de ces interfaces, notamment à l'aide de méthodes électrochimiques appliquées à des électrodes modèles et, le cas échéant, couplées à des méthodes d'analyse chimique quantitatives. Les exemples passés en revue concernent l'interphase solide-électrolyte (SEI) d'électrode négative pour accumulateur $\mathrm{Li}$-ion et la réduction électrochimique du dioxyde de carbone. Finalement, nous avons montré que certaines propriétés de ces interfaces peuvent être évaluées à l'aide de molécules redox sondes qui sont ajoutées à des fins d'analyse.

\section{Cours 6 - La batterie tout solide : l'état de l'art, les développements récents, pourquoi cet engouement soudain?}

19 mars 2018

La batterie tout solide ne cesse de faire l'objet d'annonces dans les médias de la part des constructeurs de véhicules électriques (Toyota, BMW, Renault-Nissan, ...) qui prévoient qu'en 2022, le tout solide pourrait concurrencer les véhicules Tesla. Nous avons cependant rappelé que nous avons connu le même engouement il y a de cela 5 ans, avec des annonces tout aussi optimistes sur la technologie Li-air, qui est aujourd'hui oubliée. Qu'en est-il donc du tout solide ? C'est ce à quoi ce dernier cours tentera de répondre en faisant état des avancées scientifiques récentes. Nous avons tout d'abord noté que la batterie tout solide est une histoire qui refait surface en raison d'une période d'activités intenses dans les années 1980, centrées autour des verres fluorés sulfurés, avec, en 1986, la réalisation par J.R. Akridge d'une batterie tout solide en couches minces qui dépassa les 1000 cycles. Les efforts n'ont pas perduré en raison de difficultés à maîtriser les interfaces et à trouver de meilleurs conducteurs ioniques. Grâce à la persévérance de certains groupes au fil des années, de nouvelles familles d'électrolytes (NASICON, GRENATS, PEROVSKITE, THIO-LISICON) ont été identifiées, la plus grande découverte ayant eu lieu en 2011, avec l'annonce par un groupe japonais d'un nouveau conducteur $\mathrm{Li}_{10} \mathrm{GeP}_{2} \mathrm{~S}_{12}$ (dénommé LGPS) qui présentait une conductivité de $10 \mathrm{mS} \cdot \mathrm{cm}^{-1}$. Il s'agit là d'une conductivité qui se compare favorablement avec celle des électrolytes liquides. Des substitutions chimiques récentes consistant à remplacer le germanium par du silicium et de l'étain, ainsi que le soufre par du chlore ou de l'oxygène ont permis d'obtenir des conductions 
ioniques de $30 \mathrm{mS} \cdot \mathrm{cm}^{-1}$ à $40 \mathrm{mS} \cdot \mathrm{cm}^{-1}$. Ces avancées furent doublées par des progrès au niveau de l'assemblage de batteries tout solide via l'utilisation du frittage à chaud (SPS), voire de la coulure de bandes. Grâce à des calculs de DFT et en accord avec les résultats expérimentaux, il a pu être montré que la plupart des électrolytes solides ne sont pas stables. Comme pour les électrolytes liquides, leur stabilité résulte de la cinétique lente des réactions de décomposition aux interfaces. Nous avons montré que l'utilisation d'alliages $\mathrm{Li}_{\mathrm{x}} \mathrm{Si}_{\mathrm{y}}$ ou $\mathrm{Li}_{\mathrm{x}} \mathrm{Al}$ permet de passer d'un comportement lithiophobe à un comportement lithiophile au niveau du Li et par là-même d'obtenir une meilleure interface. Enfin, quant au niveau de la sécurité, des mesures de DSC ont révélé un pic exothermique associé à la réaction $\left(2 \mathrm{Li}+1 / 2 \mathrm{O}_{2} \rightarrow \mathrm{Li}_{2} \mathrm{O}\right)$, ce qui fait que ces batteries sont également inflammables. En résumé, la batterie tout solide a fait récemment l'objet de progrès conséquents suscitant de nombreux espoirs. Ils doivent cependant être modérés par la complexité des interfaces non encore maîtrisée à ce jour. D'où l'aspect irréaliste de certaines annonces.

\section{Séminaire 6 - Contrôle de l'interface électrode/électrolyte dans les électrodes de supercondensateurs : la clé de la performance}

Patrice Simon (professeur, université Toulouse III - Paul Sabatier, CIRIMAT, CNRS, UMR 5085)

Les supercondensateurs sont des systèmes de stockage électrochimique de l'énergie qui offrent des performances intermédiaires entre les batteries et les condensateurs. Ils sont en effet capables de délivrer de fortes puissances pendant des temps de quelques dizaines de secondes, ce qui en fait des systèmes complémentaires aux batteries.

Les supercondensateurs stockent la charge par adsorption réversible des ions d'un électrolyte dans des électrodes de carbones poreux de grande surface développée $\left(>1,000 \mathrm{~m}^{2} \cdot \mathrm{g}^{-1}\right)$. L'interface entre l'électrode poreuse et l'électrolyte joue donc un rôle central dans ces systèmes, puisque c'est là que vont avoir lieu la plupart des réactions mises en jeu, à savoir la charge de la double couche électrochimique au travers de l'adsorption des ions, les échanges ioniques ou encore la désolvatation. L'importance de cette interface est encore renforcée par la dimension nanométrique des pores des électrodes de carbone, où le confinement de l'électrolyte dans ces nanopores va entraîner des effets de structurations spécifiques.

Dans cet exposé, nous avons montré comment le contrôle de l'interface électrode/ électrolyte dans des électrodes supercondensateurs a tout d'abord permis d'améliorer nos connaissances fondamentales sur l'adsorption et le transport des ions dans les milieux confinés. Nous nous sommes appuyés sur des résultats obtenus à partir de diverses techniques expérimentales, mais aussi de modélisation. D'un point de vue plus pratique, ces résultats ont permis d'améliorer la capacité de stockage des charges dans ces électrodes, permettant ainsi d'améliorer la densité d'énergie des supercondensateurs.

\section{RECHERCHE}

Nos activités se sont centrées sur l'exploitation des processus redox anioniques pour créer des matériaux énergétiques avancés non seulement pour le stockage d'énergie avec des batteries $\mathrm{Li}(\mathrm{Na})$-ion, mais aussi pour des applications de séparation de l'eau. Les faits saillants concernant la période considérée (mars 2017-mars 2018) sont triples. 
Ils enrôlent i) la découverte d'une nouvelle chimie riche en protons via l'échange d'ions $\mathrm{Li}^{+}--\mathrm{H}^{+}$dans les phases riches en $\mathrm{Li}$, ii) l'identification de $\beta-\mathrm{H}_{3} \mathrm{IrO}_{3}$ comme l'un des matériaux les plus performants pour l'électrocatalyse.

\section{JOUER AVEC L'ACTIVITÉ REDOX ANIONIQUE}

Le concept d'activité anionique a été initialement établi via des composés modèles dont la structure et les performances étaient identiques à celles de la NMC1 riche en Li. Il restait cependant à démontrer que notre mécanisme proposé pouvait s'appliquer aux phases lamellaires riches en Li (les plus convoitées actuellement pour les applications). C'est par des mesures HARD-XPS qui sondent les particules en profondeur que nous avons pu répondre positivement à cette question et définitivement montrer qu'il s'agissait bien d'un effet de cœur et non de surface (Nature Communications). Poursuivant nos études sur la phase $\mathrm{Li}_{3} \mathrm{IrO}_{4}$, nous avons par la suite montré l'importance du paramètre $\mathrm{O} / \mathrm{M}$ qui contrôle la capacité maximale du matériau, c'est-à-dire qui régle le compromis entre stabilité structurale et départ de $\mathrm{O}_{2}$. De façon surprenante, la phase homologue au $\mathrm{Ru}, \mathrm{Li}_{2} \mathrm{RuO}_{3}$, se comporte différemment avec l'absence de libération de $\mathrm{O}_{2}$ au détriment de la dégradation/ dissolution du matériau. Via l'étude du système $\mathrm{Li}_{3} \mathrm{Ru}_{-}{ }_{y} \mathrm{Ir}_{y} \mathrm{O}_{4}$, avons identifié les principaux paramètres régissant le mécanisme de dégradation du matériau lors de l'activation du processus redox anionique. De faibles quantités d'Ir $(<0, .1)$ s'avèrent suffisantes pour déclencher la libération massive de $\mathrm{O}_{2}$. Nous avons pu rendre compte de ces observations par des considérations structurales, à savoir la possibilité $\mathrm{du}$ réseau d'oxygène pour stabiliser la transformation des octaèdres $\mathrm{RuO}_{6}$ en tétraèdres $\mathrm{RuO}_{4}$ dans $\mathrm{Li}_{3} \mathrm{RuO}_{4}$ et non dans $\mathrm{Li}_{3} \mathrm{IrO}_{4}$. Ce travail a fait l'objet d'un papier dans Nature Chemistry (daté du 9 avril 2018).

\section{DÉCLENCHEMENT D'UNE NOUVELLE CHIMIE DU PROTON}

Lors de nos études sur la phase $\mathrm{Li}_{3} \mathrm{IrO}_{4}$, nous avons obtenu par réaction d'échange $\left(\mathrm{Li}^{+}--\mathrm{H}^{+}\right)$une nouvelle phase lamellaire protonnée $\mathrm{H}_{3} \mathrm{IrO}_{4}$, présentant une réactivité électrochimique vis-à-vis du $\mathrm{Li}^{+}$et du $\mathrm{H}^{+}$. La généralisation de cette approche à des composés lamellaires contenant des métaux $3 \mathrm{~d}$, $4 \mathrm{~d}$ ou $5 \mathrm{~d}$ offre de grandes opportunités pour la conception judicieuse de matériaux d'électrode attractifs pour la fabrication de batteries aqueuses rechargeables. Une telle réaction d'échange d'ions a été conduite de façon hydrothermale pour préparer la phase $3 \mathrm{D} \beta-\mathrm{H}_{2} \mathrm{IrO}_{3}$, qui s'avère être la meilleure catalyse de séparation d'eau jamais rapportée. Un brevet est en cours de dépôt.

\section{ACTIVITÉ REDOX ANIONIQUE DANS LES COMPOSÉS NA}

En parallèle, nos efforts se sont portés sur la recherche de nouvelles électrodes positives à base de $\mathrm{Na}$ à haute capacité. À la lumière de nos travaux antécédents sur la phase $\mathrm{Na}_{2} \mathrm{RuO}_{3}$, nous avons synthétisé une nouvelle phase $\beta-\mathrm{Na}_{1.7} \mathrm{IrO}_{3}$ de stucture tridimensionnelle, par un processus en deux étapes, qui implique i) l'élimination électrochimique du $\mathrm{Li}$ de $\beta-\mathrm{L}_{\mathrm{i} 2} \mathrm{IrO}_{3}$ pour produire la phase $\beta-\mathrm{IrO}_{3}$, qui est ensuite ii) réduite par l'insertion électrochimique du Na. $\beta-\mathrm{Na}_{1.7} \mathrm{IrO}_{3}$ peut absorber de manière réversible $1,3 \mathrm{Na}^{+}$, selon des processus cationiques et anioniques cumulatifs. Par la suite, de nouveaux ruthénates à base de $\mathrm{Na}$, dans 
lesquels $\mathrm{Ru}$ est $5+\left(\mathrm{Na}_{3} \mathrm{RuO}_{4}\right)$ ou $6+\left(\mathrm{Na}_{2} \mathrm{RuO}_{4}\right)$, s'avérèrent essentiaux pour comprendre les paramètres clés qui gouvernent l'oxydation du $\mathrm{R}$, plutôt que de l'oxygène lorsque ces matériaux sont oxydés électrochimiquement. Ce comportement est ancré dans la coordination du Ru comme détaillée dans la une publication dans JACS. Enfin, motivés par notre envie de fabriquer des électrodes pratiques à base de $\mathrm{Na}$, nous portons notre attention sur des phases lamellaires à base de métaux $3 \mathrm{~d}$ et nous réussissons. Une nouvelle phase $\mathrm{Na}_{0.7} \mathrm{Zn}_{0.19} \mathrm{Mn}_{0.8} \mathrm{O}_{2}$ déficitaire en $\mathrm{Na}$ présentant une activité rédox anionique et de bonnes performances en cyclage fut synthétisée. Cette découverte est quelque peu contre-intuitive, du fait que la chimie de $\mathrm{Mg}$ est différente de celle du $\mathrm{Zn}$ ( $\mathrm{MgO}$ plus ionique que $\mathrm{ZnO}$ ). Des calculs DFT sont en cours pour tenter de rendre compte d'une telle activité.

\section{THÉORIE}

La course à une plus grande capacité a conduit à tirer profit du redox cumulé du réseau cationique et du réseau anionique. Une question légitime concerne la possibilité d'étendre l'activité redox anionique aux électrodes à base de $\mathrm{Na}$. Curieusement, nous avons réussi, à la lumière des travaux précédents sur $\mathrm{Na}_{0.7} \mathrm{Mg}_{1 / 3} \mathrm{Mn}_{2 / 3} \mathrm{O}_{2}$, une nouvelle phase $\mathrm{Na}_{0.7} \mathrm{Zn}_{1 / 3} \mathrm{Mn}_{2 / 3} \mathrm{O}_{2}$ qui, tout comme la $\mathrm{Mg}$, montre une activité redox anionique alors qu'elles sont toutes deux déficitaires en $\mathrm{Na}^{+}$. Les calculs DFT ont été poursuivis pour rationaliser un tel nouveau résultat intriguant. Ces calculs montrèrent l'utilisation possible de la distorsion comme une coordonnée de réaction pour étudier le paysage énergétique du matériau pendant la dés-intercalation, offrant ainsi une méthode générale pour évaluer la stabilité structurelle d'un matériau vis-à-vis du redox anionique.

\section{ÉLECTROCATALYSE : MATÉRIAUX DE FRACTIONNEMENT DE L'EAU}

Notre groupe montra que des catalyseurs à base de structure pérovskite pouvaient grandement améliorer la cinétique de la réaction d'évolution de l'oxygène (OER) tout en déclenchant des instabilités de surface (Catalysts, 2017). Pour comprendre le rôle joué par l'oxygène oxydé en surface et ensuite stimuler la cinétique de surface, de nouvelles stratégies de revêtement furent développées. Deux faits saillants résument ces travaux. La démonstration qu'une modification de la charge d'oxygène de surface lors de l'oxydation conduit à la formation d'états de protonation qui régissent la cinétique du processus global d'évolution de l'oxygène (Angewandte, 2017). À partir de cette observation, nous avons ensuite enduit la surface de catalyseurs OER pérovskite avec des groupes phosphate ( $\mathrm{pka} \sim 12$ ), afin d'améliorer sélectivement le transfert de protons de surface, et réussi à augmenter l'activité OER de ces catalyseurs par un facteur 2-3 (JPCL, 2017). En outre, nous avons démontré que l'augmentation du $\mathrm{pH}$ est bénéfique pour améliorer la cinétique OER des catalyseurs à activité anionique redox, mais nuisible en déclenchant des réactions de dégradation telles que la corrosion et la dissolution des cations (document soumis). Nous travaillons actuellement sur de nouvelles stratégies de design pour contrebalancer ces réactions parasites. Plus précisément, nous avons pu démontrer que l'utilisation d'une structure adéquate telle que la couche $\mathrm{Li}_{2} \mathrm{IrO}_{3}$ permettait l'insertion de cations en solution ayant le rayon hydrodynamique approprié tel que $\mathrm{K}^{+}$au cours de l'OER. Une telle réduction contre-intuitive des catalyseurs sur des conditions oxydantes est en fait imbriquée dans la réaction chimique du catalyseur avec l'eau, que nous démontrons 
produire de l'oxygène à une cinétique plus grande que l'oxydation électrocatalytique traditionnelle de l'eau.

\section{TRANSFERT DE CONNAISSANCES ET DE TECHNOLOGIE}

Plusieurs aspects du travail susmentionné portant sur i) le développement d'une nouvelle méthode de détection d' $\mathrm{O}_{2}$ ou ii) l'identification d'un nouvel électrocatalyseur $\left(\mathrm{b}-\mathrm{H}_{2} \mathrm{IrO}_{3}\right)$ pour la séparation de l'eau font l'objet de brevets en cours de dépôt. Parallèlement, notre expertise cumulée ces dernières années sur le système redox anionique et principalement sur la synthèse de la phase $\mathrm{Li}_{2} \mathrm{Ru}_{1}$. ${ }_{\mathrm{x}} \mathrm{Sn}_{\mathrm{x}} \mathrm{O}_{3}$ avec une capacité de $270 \mathrm{mAh} / \mathrm{g}$ et une décroissance de tension limitée est actuellement transférée à l'ESA (European Space Agency) via un projet. Le but du projet est de développer des cellules $\mathrm{Li}_{2} \mathrm{Ru}_{1-\mathrm{x}} \mathrm{Sn}_{\mathrm{x}} \mathrm{O}_{3} / \mathrm{C}$ pour des applications spatiales, afin de quantifier la valeur ajoutée de ces nouveaux matériaux en termes d'énergie spécifique et gravimétrique dans des cellules commerciales 18650.

\section{BREVETS}

YANG C. et al., «Synthesis of new Ir-based electrocatalysts for OER reactions », déposé le 25 avril 2018.

YIN W. et al., «Use of RDDE as a precise analytical to determine the amount of $\mathrm{O}$ release in Li-rich NMC as well as Na deficient layered materials », déposé en mai 2018.

\section{PuBLications}

ASSAT G. et TARASCON J.-M., «Fundamental understanding and practical challenges of anionic redox activity in Li-ion batteries », Nature Energy, vol. 3, no 5, 2018, p. 373-386, DOI : 10.1038/s41560-018-0097-0.

Assat G., Foix D., Delacourt C., Iadecola A., Dedryvère R. et TARascon J.-M., «Fundamental interplay between anionic/cationic redox governing the kinetics and thermodynamics of lithium-rich cathodes ", Nature Communications, vol. 8, $\mathrm{n}^{\mathrm{0}} 1$, 2017, p. 2219, DOI : 10.1038/s41467-017-02291-9 [HAL : 01677634].

Assat G., Iadecola A., Delacourt C., Dedryvere R. et Tarascon J.-M., « Decoupling cationic-anionic redox processes in a model Li-rich cathode via operando X-ray absorption spectroscopy », Chemistry of Materials, vol. 29, $\mathrm{n}^{\circ}$ 22, 2017, p. 9714-9724, DOI : 10.1021/ acs.chemmater.7b03434 [HAL : 01679477].

Perez A., Jacquet Q., Batuk D., Iadecola A., Saubanère M., Rousse G., Larcher D., Vezin H., Doublet M.-L. et TARASCON J.-M., «Approaching the limits of cationic and anionic electrochemical activity with the Li-rich layered rocksalt $\mathrm{Li}_{3} \mathrm{IrO}_{4}$ ", Nature Energy, vol. 2, no 12, 2017, p. 954-962, DOI : 10.1038/s41560-017-0042-7 [HAL : 01660054].

Jacquet Q., Perez A., Batuk D., VAn Tendeloo G., Rousse G. et TARAscon J.-M., « The $\mathrm{Li}_{3} \mathrm{Ru}_{y} \mathrm{Nb}_{1-} \mathrm{O}_{4}(0 \leq y \leq 1)$ system: Structural diversity and $\mathrm{Li}$ insertion and extraction capabilities », Chemistry of Materials, vol. 29, n 12, 2017, p. 5331-5343, DOI : 10.1021/acs. chemmater.7b01511.

Perez A.J., Beer R., Lin Z., SAlager E., TAberna P.-L., Abakumov A.M., Simon P. et TARASCON J.-M., « Proton ion exchange reaction in $\mathrm{Li}_{3} \mathrm{IrO}_{4}$ : A way to new $\mathrm{H}_{3}+{ }_{x} \mathrm{IrO}_{4}$ phases electrochemically active in both aqueous and nonaqueous electrolytes », Advanced Energy Materials, vol. 8, no 13, 2018, p. 1702855, DOI : 10.1002/aenm.201702855. 
YANG C., FontAine O., TARASCON J.-M. et Grimaud A., « Chemical recognition of active oxygen species on the surface of oxygen evolution reaction electrocatalysts », Angewandte Chemie (International ed. in English), vol. 56, no 30, 2017, p. 8652-8656, DOI : 10.1002/ anie. 201701984.

YANG C. et GRIMAUD A., «Factors controlling the redox activity of oxygen in perovskites: From theory to application for catalytic reactions », Catalysts, vol. 7, no 5, 2017, p. 149, DOI : $10.3390 /$ catal7050149.

Yang C., Laberty-Robert C., Batuk D., Cibin G., Chadwick A.V., Pimenta V., Yin W., Zhang L., TARASCON J.-M. et GRIMAUD A., « Phosphate ion functionalization of perovskite surfaces for enhanced oxygen evolution reaction », The Journal of Physical Chemistry Letters, vol. 8, no 15, 2017, p. 3466-3472, DOI : 10.1021/acs.jpclett.7b01504.

Bai X., Sathiya M., Mendoza-Sánchez B., Iadecola A., Vergnet J., Dedryvère R., SAUBANÈRE M., ABAKUMOV A.M., ROZIER P. et TARASCON J.-M., « Anionic redox activity in a newly $\mathrm{Zn}$-doped sodium layered oxide $\mathrm{P} 2-\mathrm{Na}_{2 / 3} \mathrm{Mn}_{1-y} \mathrm{Zn}_{y} \mathrm{O}_{2}(0<y<0.23)$ », Advanced Energy Materials, vol. 8, no 32, 2018, p. 1802379, DOI : 10.1002/aenm.201802379.

Blazquez-Alcover I., Rousse G., Corte D.A.D., BAdot J.C., Grimaud A., Rozier P. et TARASCON J.-M., «Improving ionic conductivity by Mg-doping of $\mathrm{A}_{2} \mathrm{SnO}_{3}\left(\mathrm{~A}=\mathrm{Li}^{+}, \mathrm{Na}^{+}\right)$», Solid State Ionics, vol. 308, 2017, p. 16-21, DOI : 10.1016/j.ssi.2017.05.013 [HAL : 01529702].

Grimaud A., Iadecola A., Batuk D., Saubanère M., Abakumov A., Freeland J., Cabana J., Li H., Doublet M.-L., Rousse G. et TARAscon J.-M., « Chemical activity of the peroxide/oxide redox couple : Case study of $\mathrm{Ba}_{5} \mathrm{Ru}_{2} \mathrm{O}_{11}$ in aqueous and organic solvents », Chemistry of Materials, vol. 30, $\mathrm{n}^{\mathrm{o}}$ 11, 2018, p. 3882-3893, DOI : 10.1021/acs. chemmater.8b01372 [HAL : 01825124].

Grimaud A., Yin W., LePoivre F. et TARAscon J.-M., «Controlling the specific $\mathrm{CO}_{2}$ adsorption on electrochemically formed metallic copper surfaces », Journal of The Electrochemical Society, vol. 165, no 3, 2018, p. H163-H169, DOI : 10.1149/2.0031805jes.

Jacquet Q., Iadecola A., Saubanère M., Lemarquis L., Berg E.J., Alves Dalla Corte D., Rousse G., Doublet M.-L. et Tarascon J.-M., " Competition between metal dissolution and gas release in Li-rich $\mathrm{Li}_{3} \mathrm{Ru}_{y} \mathrm{Ir}_{1}-{ }_{y} \mathrm{O}_{4}$ model compounds showing anionic redox », Chemistry of Materials, vol. 30, $\mathrm{n}^{\mathrm{o}}$ 21, 2018, p. 7682-7690, DOI : 10.1021/acs. chemmater.8b02955.

Jacquet Q., Rousse G., Iadecola A., Saubanère M., Doublet M.-L. et TARascon J.-M., «Electrostatic interactions versus second order Jahn-Teller distortion as the source of structural diversity in $\mathrm{Li}_{3} \mathrm{MO}_{4}$ compounds $(\mathrm{M}=\mathrm{Ru}, \mathrm{Nb}, \mathrm{Sb}$ and $\mathrm{Ta})$ », Chemistry of Materials, vol. 30, n 2, 2018, p. 392-402, DOI : 10.1021/acs.chemmater.7b04117 [HAL : 01692211].

LANDER L., TARASCON J.-M. et YAMADA A., «Sulfate-based cathode materials for Li- and Na-ion batteries », The Chemical Record, vol. 18, no 10, 2018, p. 1394-1408, DOI : 10.1002/ tcr.201800071.

Lutz L., Alves Dalla Corte D., Tang M., Salager E., Deschamps M., Grimaud A., Johnson L., BRUCE P.G. et TARASCON J.-M., «Role of electrolyte anions in the $\mathrm{Na}-\mathrm{O}_{2}$ battery : Implications for $\mathrm{NaO}_{2}$ solvation and the stability of the sodium solid electrolyte interphase in glyme ethers », Chemistry of Materials, vol. 29, $\mathrm{n}^{\circ}$ 14, 2017, p. 6066-6075, DOI : 10.1021/acs.chemmater.7b01953.

Lutz L., Corte D.A.D., Chen Y., Batuk D., Johnson L.R., Abakumov A., Yate L., AZACETA E., BRUCE P.G., TARASCON J.-M. et GRIMAUD A., « The role of the electrode surface in $\mathrm{Na}$-Air batteries : Insights in electrochemical product formation and chemical growth of $\mathrm{NaO}_{2}$ », Advanced Energy Materials, vol. 8, no 4, 2018, p. 1701581, DOI : 10.1002/aenm.201701581.

Lutz L., Dachraoui W., Demortière A., Johnson L.R., Bruce P.G., Grimaud A. et TARASCON J.-M., «Operando monitoring of the solution-mediated discharge and charge processes in a $\mathrm{Na}-\mathrm{O}_{2}$ battery using liquid-electrochemical transmission electron microscopy », Nano Letters, vol. 18, n 2, 2018, p. 1280-1289, DOI : 10.1021/acs.nanolett.7b04937. 
MARIYAPPAN S., WANG Q. et TARASCON J.M., «Will sodium layered oxides ever be competitive for sodium ion battery applications? », Journal of the Electrochemical Society, vol. 165, no 16, 2018, p. A3714, DOI : 10.1149/2.0201816jes.

Parant H., Muller G., Le Mercier T., Tarascon J.M., Poulin P. et Colin A., «Flowing suspensions of carbon black with high electronic conductivity for flow applications: Comparison between carbons black and exhibition of specific aggregation of carbon particles », Carbon, vol. 119, no 4, 2017, p. 10-20, DOI : 10.1016/j.carbon.2017.04.014 [HAL : 01544046].

Parant H., Muller G., Mercier T.L., Poulin P., Tarascon J.-M. et Colin A., « Complete study of a millifluidic flow battery using iodide and ferricyanide ions : modeling, effect of the flow and kinetics », Microfluidics and Nanofluidics, vol. 21, $\mathrm{n}^{\circ}$ 11, 2017, p. 171, DOI : 10.1007/s10404-017-2009-1 [HAL : 01688085].

Pearce P., Rousse G., Karakulina O., Hadermann J., Van Tendeloo G., Foix D., FAUTH F., ABAKUMOV A. et TARASCON J.-M., « $\beta-\mathrm{Na}_{1.7} \mathrm{IrO}_{3}$ : A tridimensional Na-ion insertion material with a redox active oxygen network », Chemistry of Materials, vol. 30, $n^{\mathrm{o}} 10$, 2018, p. 3285-3293, DOI : 10.1021/acs.chemmater.8b00320 [HAL : 01802481].

Pimenta V., Sathiya M., Batuk D., Abakumov A.M., Giaume D., Cassaignon S., LARCHER D. et TARASCON J.-M., «Synthesis of Li-rich NMC: A comprehensive study », Chemistry of Materials, vol. 29, $\mathrm{n}^{\circ}$ 23, 2017, p. 9923-9936, DOI : 10.1021/acs.chemmater.7b03230.

Rousse G., Ahouari H., Pomjakushin V., TARascon J.-M., Recham N. et AbaKumov A.M., «Denticity and mobility of the carbonate groups in AMCO3F fluorocarbonates: A Study on $\mathrm{KMnCO} 3 \mathrm{~F}$ and High Temperature $\mathrm{KCaCO}_{3} \mathrm{~F}$ polymorph », Inorganic Chemistry, vol. $56, \mathrm{n}^{\circ} 21$, 2017, p. 13132-13139, DOI : 10.1021/acs.inorgchem.7b01926.

Saha S., Rousse G., Alcover I.B., Courty M., Corte D.A.D. et TARascon J.-M., «Polymorphism in $\mathrm{Li}_{4} \mathrm{Zn}\left(\mathrm{PO}_{4}\right)_{2}$ and stabilization of its structural disorder to improve ionic conductivity », Chemistry of Materials, vol. 30, no 4, 2018, p. 1379-1390, DOI : 10.1021/acs. chemmater.7b05139 [HAL : 01778015].

Sathiya M., Jacquet Q., Doublet M.-L., Karakulina O., Hadermann J. et TARASCON J.-M., «A chemical approach to raise cell voltage and suppress phase transition in $\mathrm{O}_{3}$ sodium layered oxide electrodes », Advanced Energy Materials, vol. 8, n ${ }^{\circ}$ 11, 2018, p. 1702599, [HAL : 01692208].

Sathiya M., Thomas J., Batuk D., Pimenta V., Gopalan R. et Tarascon J.-M., « Dual stabilization and sacrificial effect of $\mathrm{Na}_{2} \mathrm{CO}_{3}$ for increasing capacities of Na-ion cells based on P2- $\mathrm{Na}_{x} \mathrm{MO}_{2}$ electrodes », Chemistry of Materials, vol. 29, $\mathrm{n}^{\mathrm{o}} 14$, 2017, 5948-5956, [HAL : 01557319].

SimON P. et TARASCON J.-M., "Apports de la chimie pour de meilleurs accumulateurs », in C. JEANDEL et R. MOSSERI (dir.), L'Énergie à découvert, Paris, CNRS Éditions, coll. « À découvert », 2017, p. 202-203, DOI : 10.4000/books.editionscnrs.11101 ; en ligne : http:// books.openedition.org/editionscnrs/11101.

Strauss F., Rousse G., Alves Dalla Corte D., Giacobbe C., Dominko R. et TARASCON J.-M., « Impact of structural polymorphism on ionic conductivity in lithium copper pyroborate $\mathrm{Li}_{6} \mathrm{CuB}_{4} \mathrm{O}_{10}$ », Inorganic Chemistry, vol. 57, $\mathrm{n}^{\circ}$ 18, 2018, p. 11646-11654, DOI : 10.1021/acs.inorgchem.8b01785.

Strauss F., Rousse G., Batuk D., TAng M., SAlager E., Dražić G., Dominko R. et TARASCON J.-M., «Electrochemical behavior of $\mathrm{Bi}_{4} \mathrm{~B}_{2} \mathrm{O}_{9}$ towards lithium-reversible conversion reactions without nanosizing », Physical chemistry chemical physics, PCCP, vol. 20, n 4, 2018, p. 2330-2338, DOI : 10.1039/c7cp07693b [HAL : 01739762].

TARAsCon J.-M., « Chimie du solide et énergie », Annuaire du Collège de France 2015-2016. Résumé des cours et travaux, $\mathrm{n}^{\circ} 116,2018$, p. 87-102, DOI : 10.4000/annuaire-cdf.12802 ; en ligne : https://journals.openedition.org/annuaire-cdf/12802. 
Westman K., Dugas R., Jankowski P., Wieczorek W., Gachot G., Morcrette M., Irisarri E., Ponrouch A., PAlacín M.R., TARAscon J.-M. et Johansson P., « Diglyme based electrolytes for sodium-ion batteries », ACS Applied Energy Materials, vol. 1, $\mathrm{n}^{\circ}$ 6, 2018, p. 2671-2680, DOI : 10.1021/acsaem.8b00360.

YAN G., DUGAS R. et TARASCON J.-M., «The $\mathrm{Na}_{3} \mathrm{~V}_{2}\left(\mathrm{PO}_{4}\right) 2 \mathrm{~F} 3 /$ carbon Na-ion battery : Its performance understanding as deduced from differential voltage analysis », Journal of the Electrochemical Society, vol. 165, no 2, 2018, p. A220-A227, DOI : 10.1149/2.0831802jes.

Yan G., Alves-Dalla-Corte D., Yin W., Madern N., Gachot G. et Tarascon J.-M., "Assessment of the electrochemical stability of carbonate-based electrolytes in Na-ion batteries », Journal of The Electrochemical Society, vol. 165, no 7, 2018, p. A1222-A1230, DOI : 10.1149/2.0311807jes.

Yang C., Batuk M., Jacquet Q., Rousse G., Yin W., Zhang L., Hadermann J., AbaKumov A.M., Cibin G., ChadWick A., TARASCON J.-M. et Grimaud A., « Revealing $\mathrm{pH}$-dependent activities and surface instabilities for Ni-based electrocatalysts during the oxygen evolution reaction », ACS Energy Letters, vol. 3, n 12, 2018, p. 2884-2890, [HAL : 01958610].

Yin W., Grimaud A., Azcarate I., Yang C. et TARAscon J.-M., « Electrochemical reduction of $\mathrm{CO}_{2}$ mediated by quinone derivatives : Implication for $\mathrm{Li}-\mathrm{CO}_{2}$ battery », The Journal of Physical Chemistry C, vol. 122, no 12, 2018, p. 6546-6554, DOI : 10.1021/acs. jpcc.8b00109.

Yin W., MARIYAPPAN S., GRIMAUD A. et TARASCON J.M., « Rotating ring disk electrode for monitoring the oxygen release at high potentials in Li-rich layered oxides », Journal of The Electrochemical Society, vol. 165, no 14, 2018, p. A3326-A3333, DOI : 10.1149/2.0481814jes.

Zhang L., Dambournet D., Iadecola A., Batuk D., Borkiewicz O.J., WiadereK K.M., SAlager E., Shao M., ChEN G. et TARAsCON J.-M., « Origin of the high capacity manganesebased oxyfluoride electrodes for rechargeable batteries », Chemistry of Materials, vol. 30, $n^{0} 15,2018$, p. 5362-5372, DOI : 10.1021/acs.chemmater.8b02182. 
